\title{
On the Performance of Different Simulation Models for Rayleigh Fading Channels
}

\author{
Mohamed Ali Skima ${ }^{1}$, Amira Boulaaba ${ }^{2}$, Hamadi Ghariani ${ }^{1}$ \\ ${ }^{1}$ Laboratory of Electronics and Information Technology (LETI), National School of Engineers of Sfax, \\ University of Sfax, B.P. 1173, 3038 Sfax, Tunisia. \\ 2 Department of Electrical Engineering, Higher Institute of Technological Studies of Rades, Tunis, Tunisia. \\ * Corresponding author. Email: mohamedali.skima@gmail.com \\ Manuscript submitted August 3, 2015; accepted October 25, 2015. \\ doi: 10.17706/ijcce.2016.5.4.229-237
}

\begin{abstract}
In this paper, we analyze and compare seven of the most accepted and approved simulation models for Rayleigh fading channels. These models cover the three popular methods for generating time-correlated Rayleigh fading processes: the inverse discrete Fourier transform (IDFT) method, the filtering white Gaussian noise (FWGN) method and the sum-of-sinusoids (SOS) method. Three quantitative measures are used for judicious assessment of the quality of generated processes. The study addresses firstand second-order statistics of the fading process. The variance of the autocorrelation function (ACF) of the fading process is used to investigate the convergence of the stochastic SOS-based models. An evaluation of the computational effort is additionally presented. The simulation results suggest that the recently published Wang's model is very attractive and it outperforms all SOS models. This study leads to several important conclusions about the accuracy, capability and efficiency of the different Rayleigh fading generators.
\end{abstract}

Key words: Radio propagation channels, Clarke's model, Rayleigh fading channel simulators, performance analysis.

\section{Introduction}

In the case of mobile radio systems operating in typical urban areas, the envelope fluctuations of the low-pass equivalent received signal are often modeled by a random process with Rayleigh distribution and time-correlated samples [1]. This result follows from the well-known Clarke's reference model [2] for frequency non-selective mobile radio channels. Despite the widespread acceptance of Clarke's reference model, it is considered as computationally inefficient [3]. Consequently, many computer simulation models and algorithms have been proposed to overcome this problem and to simulate the Rayleigh fading process in an efficient manner. These models and algorithms are mainly classified into three methods: the inverse discrete Fourier transform (IDFT) method [4], the filtering white Gaussian noise (FWGN) method [5] and the Sum-Of-Sinusoids (SOS) method [3], [6]-[10]. Each method has encountered several modifications and improvements over time. This gives rise to numerous models and techniques for the generation of time-correlated Rayleigh processes. Unfortunately, a little work has been reported on the comparison between these models. Furthermore, the evaluation of different Rayleigh fading models is generally made through qualitative methods via a visual inspection of some statistical quantities plots and the assessment based on quantitative measures is rarely investigated. More particularly, many authors have recently 
presented new models and have concluded that their models are efficient and accurate, but further studies are needed to confirm or deny these statements.

This paper compares and analyzes the performance of the most accepted Rayleigh fading channel simulators, and it is a continuation of our previous work [11] with substantial novel contributions. Some important outcomes of [11] are reported here, to be further studied and discussed. We will use three quantitative measures to investigate the accuracy of different Rayleigh fading generators in terms of both first- and second-order statistics. In [11], the quality of the second-order time-averaged autocorrelation function (ACF) is investigated using the power margin quality measures [12]. For the non-ergodic stochastic simulation models, this statistic varies from one simulation trial to another and a look into the convergence of these models is highly recommended which is missing in [11]. The convergence of two of the most successful stochastic SOS-based models is checked here using the variance of time-averaged ACF. In addition, the first-order statistic probability density function (PDF) of the envelope is assessed with a more convenient measure than used in previous studies [11], [13]. Moreover, the complexity of the different models is tested based on the time of execution. Compared to [11], the method with which the time of execution was computed has been refined for more accurate results.

The rest of this paper is organized as follows. Section 2 presents the statistical properties of the Clarke's reference model and provides the Rayleigh fading models that were adopted in our comparative study. The simulation results and discussions are presented in section 3. Finally, section 4 concludes the paper.

\section{The Reference Model and Simulation Models}

Under the frequency nonselective fading assumption and considering the Clarke's two-dimensional (2-D) isotropic scattering model, the low-pass fading process $g(t)=g_{I}(t)+j g_{Q}(t)$, in densely built-up areas, has been shown to be approximated by a complex wide sense stationary (WSS) Gaussian random process [17]. The in-phase component $g_{I}(t)=\mathfrak{R}\{g(t)\}$, and the quadrature component $g_{Q}(t)=\mathfrak{J}\{g(t)\}$ are independent, identically distributed zero-mean Gaussian processes with identical variances $\sigma^{2}$. Thereby, the envelope $|g(t)|$ follows the well-known Rayleigh distribution with the corresponding PDF given by [2], [14]:

$$
f_{|g|}(r)=\left(r / \sigma^{2}\right) \exp \left(-r^{2} / 2 \sigma^{2}\right)
$$

Adopting the above assumptions and considering the Jakes power spectral density (PSD), the normalized $\left(\sigma^{2}=1\right)$ correlation properties of the fading process are [2], [14]:

$$
\begin{gathered}
R_{g_{I} g_{I}}(\tau)=E\left[g_{I}(t) g_{I}(t+\tau)\right]=\mathcal{J}_{0}\left(\omega_{d} \tau\right) \\
R_{g_{Q} g_{Q}}(\tau)=\mathcal{J}_{0}\left(\omega_{d} \tau\right) \\
R_{g_{I} g_{Q}}(\tau)=R_{g_{Q} g_{I}}(\tau)=0 \\
R_{g g}(\tau)=E\left[g(t) g^{*}(t+\tau)\right]=2 \mathcal{J}_{0}\left(\omega_{d} \tau\right)
\end{gathered}
$$

where $E$ is the statistical expectation operator, $\mathcal{J}_{0}(\cdot)$ is the zeroth-order Bessel function of the first kind, $\tau$ is the time lag and $\omega_{d}=2 \pi f_{d}$ is the maximum radian Doppler frequency due to the receiver motion. $R_{g_{I} g_{I}}(\tau)$ and $R_{g_{Q} g_{Q}}(\tau)$, are the ACFs of the in-phase and quadrature components of $g(t)$, respectively,

$R_{g_{I} g_{Q}}(\tau)$ and $R_{g_{Q} g_{I}}(\tau)$ are the cross-correlation functions (CCFs) of these components and $R_{g g}(\tau)$ is the 
ACF of the low-pass complex fading process $g(t)$. These results characterize completely the reference model established by Clarke. Hence, any Rayleigh fading simulator aims to reproduce the aforementioned statistical properties as faithfully as possible.

In the following, we provide the Rayleigh fading simulation models which will be used later in our study. Totally, seven of the most accepted and approved models were investigated and they are summarized in Table 1. These models are chosen carefully based on earlier works [8], [9], [11], [15] and they cover the three common methods for generating time-correlated Rayleigh fading. Details of these models are not provided here due to space constraints, but we refer the reader to the corresponding references. For the SOS method, the principle consists in approximating each of the Gaussian processes $g_{I}(t)$ and $g_{Q}(t)$ forming $g(t)$ by a finite number of sinusoids with amplitudes, frequencies and phases that are properly selected to fit the desired statistical properties. Here, the number of sinusoids used in the in-phase and quadrature branches are denoted $N_{I}$ and $N_{Q}$ respectively. Also, two classes of SOS-based model are distinguished: the Frequency-Amplitude (FA) deterministic and stochastic models [15]. In the FA-deterministic models, the sinusoids amplitudes and frequencies are deterministic. The corresponding process is ergodic, and then a single simulation trial is generally sufficient for convergence. For the stochastic models, the sinusoids amplitudes and / or frequencies are selected randomly. The statistical properties of such models vary from each simulation trial to another, and one has to compute these properties by averaging over several simulation trials which increases the complexity of the model, but helps to improve the quality. Further details about the SOS method can be found in [9].

Table 1. The Seven Rayleigh Fading Simulation Models Retained for the Comparative Study

\begin{tabular}{ccccc}
\hline \hline Model Name & Authors (Year) & Method & Reference & Notes \\
\hline Young's model & Young and Beaulieu (2000) & IDFT & {$[4]$} & - \\
AR model & Baddour and Beaulieu (2005) & FWGN & {$[5]$} & - \\
MEDS & Pätzold et al. (1996) & SOS-deterministic & [9, pp. 128-133] & $N_{Q}=N_{I}+1$ \\
ZX-1 & Zheng and Xiao (2002) & SOS-stochastic & {$[7]$} & $N_{I}=N_{Q}$ \\
M-ZX-2 & Patel et al. (2005) & SOS-stochastic & {$[8]$} & $N_{I}=N_{Q}$ \\
ZX-3 & Xiao et al. (2006) & SOS-stochastic & {$[3]$} & $N_{I}=N_{Q}$ \\
WANG & Wang et al. $(2012)$ & SOS-stochastic & {$[10]$} & $N_{I}=N_{Q}$ \\
\hline \hline
\end{tabular}

\section{Comparative Study and Simulation Results}

\subsection{Comparison Based on Power Margin Quality Measures}

In our previous work [11], we have used two quantitative measures to analyze the quality of the ACF of the real part of the seven aforementioned models. The first measure, called the mean power margin, is given by [12]:

$$
G_{\text {mean }}=\left(1 / \sigma_{x}^{2} L\right) \operatorname{trace}\left\{C_{x} C_{\hat{x}}^{-1} C_{x}\right\}
$$

The second is the maximum power margin and is defined as [12]:

$$
G_{\max }=\left(1 / \sigma_{x}^{2}\right) \max \left\{\operatorname{diag}\left\{C_{x} C_{\hat{x}}^{-1} C_{x}\right\}\right\}
$$

where, $\sigma_{x}^{2}$ is the variance of the reference (ideal) distribution, $C_{\hat{x}}$ is the $L \times L$ covariance matrix of any length- $L$ subset of adjacent samples produced by a stationary random sequence generator, and $C_{x}$ represents the desired covariance matrix of $L$ ideally distributed samples. $G_{\text {mean }}$ and $G_{\text {max }}$ measure the 
similarity of the theoretical and simulated ACFs. This second-order statistic is with high-importance because it reflects the time variability of the wireless fading channel. In all cases, the desired covariance matrix $C_{x}$ was obtained using the reference ACF of (2a), whereas the covariance matrix $C_{\hat{x}}$ was estimated using the empirical technique presented in [16, p. 545-6]. An autocorrelation sequence length of 200 was considered, at a normalized maximum Doppler frequency $f_{m}=f_{d} T$ of 0.05 , where $T$ is the sampling interval. The time average correlations needed for estimating the matrix $C_{\hat{x}}$ were calculated based on $2^{20}$ generated samples and $\sigma_{x}^{2}$ was set to unity. The computed quality measures were then averaged over 50 independent simulation trials. Generally, these measures are expressed in $\mathrm{dB}$ and a perfect performance corresponds to $0 \mathrm{~dB}$ for both quantities. The result of this study is reported here in the Table 2 [11].

Table 2. Power Margin Quality Measures of the Different Models (Reprinted from [11], with Permission from Elsevier)

\begin{tabular}{|c|c|c|c|c|c|}
\hline Method & & ame & & $G_{\text {mean }}(\mathrm{dB})$ & $G_{\max }(\mathrm{dB})$ \\
\hline IDFT & Young's model & & & 0.003129 & 0,003330 \\
\hline \multirow{3}{*}{ FWGN } & \multirow{3}{*}{ AR model } & \multirow{3}{*}{ 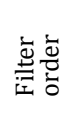 } & 50 & 0.284074 & 0.414622 \\
\hline & & & 120 & 0.050733 & 0.143090 \\
\hline & & & 200 & 0.003376 & 0.003530 \\
\hline \multirow{20}{*}{ SOS } & \multirow{4}{*}{ ZX-1 } & \multirow{4}{*}{$N_{I}$} & 8 & 34.86758 & 35.98688 \\
\hline & & & 16 & 4.931524 & 6.804558 \\
\hline & & & 64 & 0.005751 & 0.005914 \\
\hline & & & 128 & 0.001052 & 0.001092 \\
\hline & \multirow{4}{*}{ M-ZX-2 } & \multirow{4}{*}{$N_{I}$} & 8 & 38.60137 & 40.34800 \\
\hline & & & 16 & 20.64050 & 24.36247 \\
\hline & & & 64 & 0.080284 & 0.141962 \\
\hline & & & 128 & 0.012520 & 0.021570 \\
\hline & \multirow{4}{*}{$\mathrm{ZX}-3$} & \multirow{4}{*}{$N_{I}$} & 8 & 39.95607 & 41.85942 \\
\hline & & & 16 & 28.13874 & 32.17058 \\
\hline & & & 64 & 0.299641 & 0.467291 \\
\hline & & & 128 & 0.039134 & 0.064304 \\
\hline & \multirow{4}{*}{ WANG } & \multirow{4}{*}{$N_{I}$} & 8 & 33.43456 & 34.30927 \\
\hline & & & 16 & 0.154066 & 0.490900 \\
\hline & & & 64 & 0.003151 & 0.003278 \\
\hline & & & 128 & 0.000838 & 0.000871 \\
\hline & \multirow{4}{*}{ MEDS } & \multirow{4}{*}{$N_{I}$} & 8 & 33.83460 & 34.66510 \\
\hline & & & 16 & 0.153808 & 1.903978 \\
\hline & & & 64 & $2.36 \cdot 10^{-5}$ & $2.86 \cdot 10^{-5}$ \\
\hline & & & 128 & $2.70 \cdot 10^{-5}$ & $3.21 \cdot 10^{-5}$ \\
\hline
\end{tabular}

The results of Table 2 show that the accuracy of SOS-based models is strongly related to the number of sinusoids in use, $N_{I}$. Increasing $N_{I}$ leads to accurate results, but this improvement in the quality is done at the detriment of efficiency. Generally, $N_{I}=16$ is considered as a moderate choice for a good compromise between complexity and convergence. The major outcome of this study concerns the recently published WANG model. Compared with all SOS stochastic models, the WANG provides the best results in terms of $G_{\text {mean }}$ and $G_{\text {max }}$. This model outperforms the ZX-1 model considered in some comparative studies as the best stochastic model [8], [15]. Moreover, for large $N_{I}\left(N_{I} \geq 64\right)$, it outperforms the IDFT method that is always considered as accurate and efficient. On other hand, for small $N_{I}\left(N_{I}=8\right)$, the WANG model gives better results than MEDS, known by its quasi-optimal approximation of the ACF of (2a) [9]. Despite ZX-3 and M-ZX-2 have been introduced after the ZX-1 model, they failed to achieve the performances reached by the latter model. The ZX-1 stands as the best model among all Zheng and Xiao's models followed by the M-ZX-2. Additionally, the results confirm the good autocorrelation properties of the MEDS. This method gives the best autocorrelation properties among all methods provided that a sufficient number of sinusoids 
are used. Finally, the AR model of [5] has proven to be quite precise if high order model is chosen. For example, if a 200 order AR model (or higher) is taken, we can achieve a power margin quantities similar to those obtained with the IDFT method.

The results of Table 2 concern the performance evaluation of different models, in terms of the ACF of in-phase component $R_{g_{I} g_{I}}(\tau)$ of the complex process $g(\tau)$. This is completely different from evaluating the accuracy of the ACF of complex process $R_{g g}(\tau)$. This statement is confirmed by the results shown in Fig. 1(a), where we have plotted the ACF of the complex process of reference model in comparison with the ACFs of the complex process of ZX-1, WANG and MEDS models for $N_{I}=16$. Although MEDS and WANG are shown to perform similar results in terms of $R_{g_{I} g_{I}}(\tau)$, the WANG model outperforms by far the MEDS regarding the ACF of the complex process $R_{g g}(\tau)$. It can be seen that for WANG model, the range over which the simulated ACF, $R_{g g}(\tau)$ closely matches the desired one, is roughly twice wider than that of MEDS. Approximately, similar results are found for MEDS and ZX-1 models. To better conclude on the correlation properties of ZX-1, WANG and MEDS models, we have plotted in Fig. 1(b) the CCFs of the quadrature components of these models in comparison with the CCF of the quadrature components of the reference model $R_{g_{I} g_{Q}}(\tau)$. We can see clearly that the WANG model outperforms other SOS models and the CCF of its quadrature components stills near zero as desired.

(a)

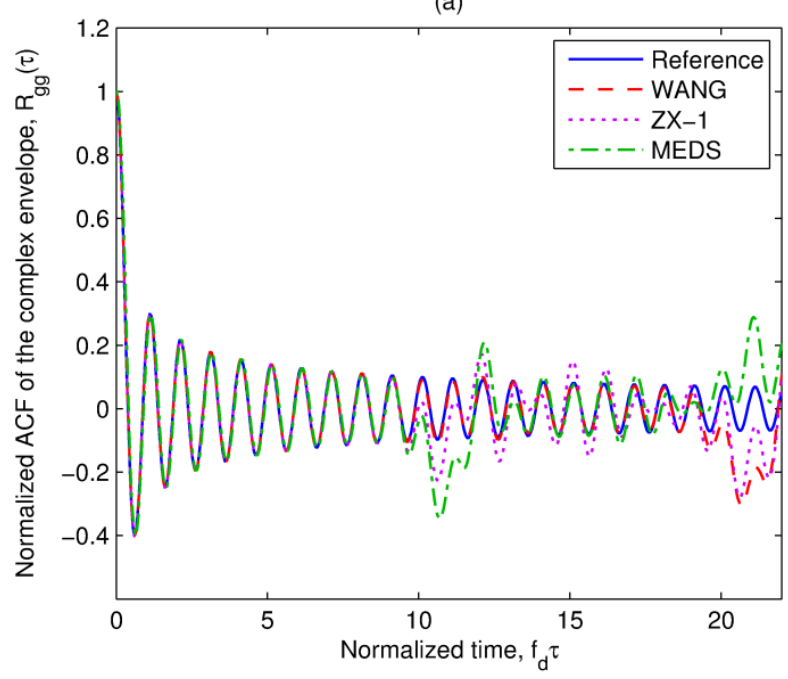

(b)

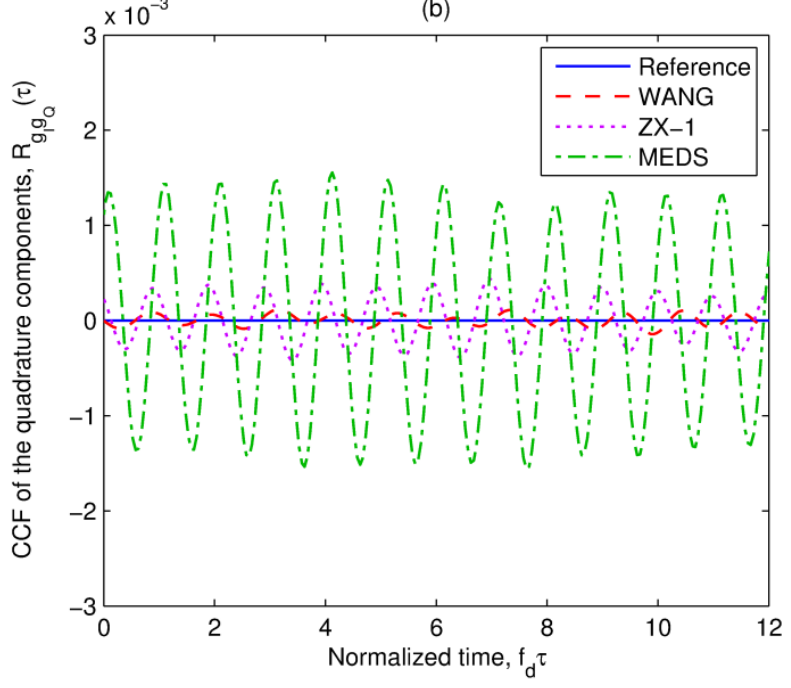

Fig. 1. (a) ACFs of the complex process $R_{g g}(\tau)$ of reference, WANG, ZX-1 and MEDS models. (b) CCFs of the quadrature components of reference, WANG, ZX-1, and MEDS models $\left(f_{m}=0.05\right.$ and $\left.N_{I}=16\right)$.

The above correlations were computed based on time-averaging, a technique that is often used in simulation practice instead of ensemble averaging [3]. For SOS stochastic models, the random frequencies makes the time-average correlation functions, denoted by $\hat{R}(\cdot)$, to change from one simulation trial to another. To characterize this variability, we generally use the variance of the time-average $\operatorname{Var}[R(\cdot)]=$ $E\left[\left|\hat{R}(\cdot)-\lim _{N_{I} \rightarrow \infty} R(\cdot)\right|^{2}\right]$ which provides important information concerning the closeness between a single trial with finite $N_{I}$ and the ideal case with $N_{I}=\infty$. A lower variance indicates that a smaller number of simulation trials are needed to achieve desired statistical properties, and hence the convergence is better. Fig. 2 shows the variances of the ACF of the complex process for ZX-1 and WANG models. We mention that these variances are computed by averaging over 500 simulation trials of a fading process with $10^{5}$ samples of length. Once again, the WANG model exhibits the better performance, and the variance of its ACF of the complex process is close to zero until a range that is roughly two times greater than the corresponding 
range of ZX-1 model. Therefore the WANG model converges faster than the ZX-1 model and its correlation properties vary little from one simulation trial to another.

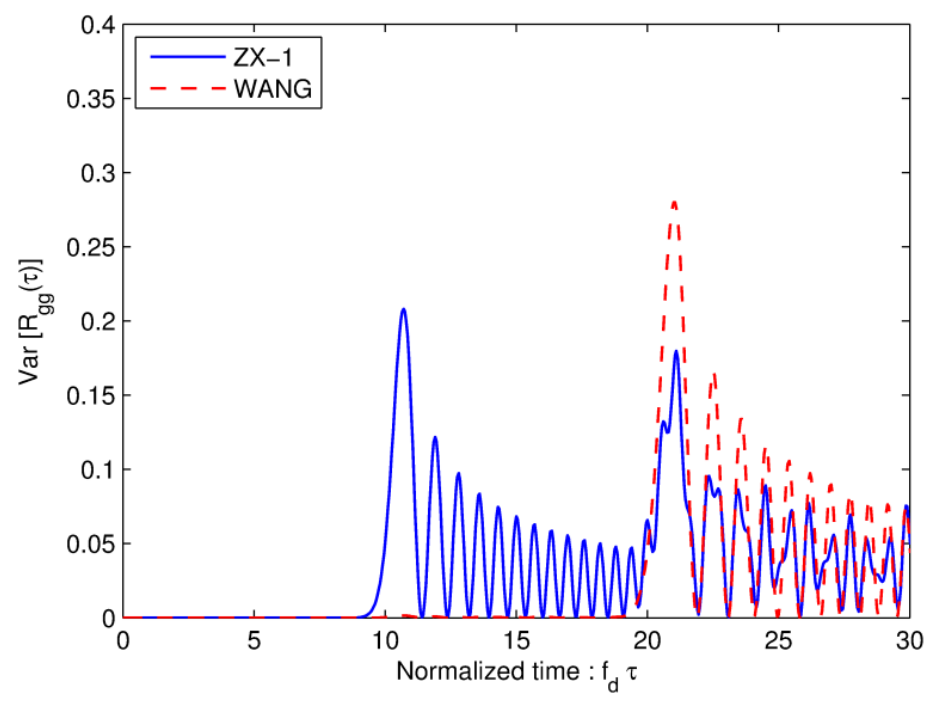

Fig. 2. Variance of ACF of the complex process $R_{g g}(\tau)$ of ZX-1 and WANG models for $f_{m}=0.05$ and $N_{I}=16$.

Table 3. Evaluation Based on the J-div of Various Models

\begin{tabular}{|c|c|c|c|c|}
\hline Model name & \multicolumn{4}{|c|}{ Method } \\
\hline \multirow[b]{2}{*}{ Young's model } & \multicolumn{4}{|c|}{ IDFT method } \\
\hline & \multicolumn{4}{|c|}{$5.742 \cdot 10^{-6}$} \\
\hline \multirow{7}{*}{ Baddour's AR model } & \multicolumn{4}{|c|}{ FWGN method } \\
\hline & \multicolumn{4}{|c|}{ Filter order } \\
\hline & 50 & \multicolumn{2}{|c|}{120} & 200 \\
\hline & $6.6 \cdot 10^{-6}$ & \multicolumn{2}{|c|}{$5.179 \cdot 10^{-6}$} & $5.712 \cdot 10^{-6}$ \\
\hline & \multicolumn{4}{|c|}{ SOS method } \\
\hline & \multicolumn{4}{|c|}{ Number of sinusoids, $N_{I}$} \\
\hline & 8 & 16 & 64 & 128 \\
\hline ZX-1 & $2.922 \cdot 10^{-3}$ & $6.209 \cdot 10^{-4}$ & $3.784 \cdot 10^{-5}$ & $1.437 \cdot 10^{-5}$ \\
\hline M-ZX-2 & $5.813 \cdot 10^{-3}$ & $1.17 \cdot 10^{-3}$ & $5.958 \cdot 10^{-5}$ & $1.876 \cdot 10^{-5}$ \\
\hline ZX-3 & $5.848 \cdot 10^{-3}$ & $1.139 \cdot 10^{-3}$ & $6.717 \cdot 10^{-5}$ & $1.926 \cdot 10^{-5}$ \\
\hline WANG & $2.996 \cdot 10^{-3}$ & $6.015 \cdot 10^{-4}$ & $4.025 \cdot 10^{-5}$ & $1.210 \cdot 10^{-5}$ \\
\hline MEDS & $2.068 \cdot 10^{-3}$ & $6.302 \cdot 10^{-4}$ & $4.742 \cdot 10^{-4}$ & $5.424 \cdot 10^{-4}$ \\
\hline
\end{tabular}

\subsection{Comparison Based on Jeffrey's Divergence Criterion}

Here, we evaluate the quality of the generated processes in terms of their envelope PDF. We use the Jeffrey's divergence (J-div) criterion commonly adopted in probability theory and statistics. This criterion measures the difference between a theoretical PDF and an empirical one. Some previous works (e.g., [11], [13]), have used the Kullback-Leibler Divergence (KL-div) criterion which is not symmetric. The J-div is the symmetric version of the KL-div criterion, and it is more convenient in this kind of studies. The J-div, denoted $D_{J}$, is defined as follows $[17$, p. 251]:

$$
D_{J}(P \| Q)=\sum_{i}(P(i)-Q(i)) \ln (P(i) / Q(i))
$$


where $P(i)$ and $Q(i)$ are the time-discrete versions of reference and the evaluated distributions respectively. The J-div is always non-negative and the closer $D_{J}$ is to 0 , the better is the agreement of the empirical PDF with the theory. All the PDFs of the envelopes of the seven Rayleigh fading simulators are evaluated using this criterion and the results of simulation are shown in Table 3. The reference PDF is given by (1). The empirical (simulated) PDFs are computed under the same condition as for $G_{\text {mean }}$ and $G_{\max }$ (i.e., $2^{20}$ samples and $f_{m}=0.05$ ). For each Rayleigh generator, the envelope PDF was averaged over 100 independent simulation trials before to be used in the J-div criterion.

According to Table 3, the IDFT and FWGN methods give the best approximation of the desired envelope PDF. For the AR model, increasing the filter order above 50 don't bring a significant improvement in the quality of the envelope PDF. The simulation results suggest that the SOS models also provide good results if a large number of sinusoids is used $\left(N_{I} \geq 64\right)$. Overall speaking, we conclude that ZX-1, WANG and MEDS are best SOS-based models regarding the J-div criterion. For small $N_{I}$, the MEDS surpasses ZX-1 and WANG. While, for moderate to large $N_{I}$, the latter models performs better than MEDS. Also, we remark that in all cases, the WANG and ZX-1 models achieve approximately similar performances. Eventually, the worst case is observed for the M-ZX-2 and ZX-3 models with a slightly better performance for the M-ZX-2 model.

\subsection{Comparison Based on the Time of Execution}

In this subsection, we evaluate the seven Rayleigh fading models in term of the computational effort. For each model, we have computed the time needed to generate 106 samples of the fading process $g(t)$ on an Intel ${ }^{\circledR}$ Core $^{\mathrm{TM}}$ i5 machine using routines coded in MATLAB $®$. The normalized maximum Doppler frequency was set equal to 0.05 . To obtain more precise results, the time of execution of each model has been averaged over 30 simulation trials. Also the outcome of the first simulation run was rejected. The results of this test are displayed in Fig. 3.
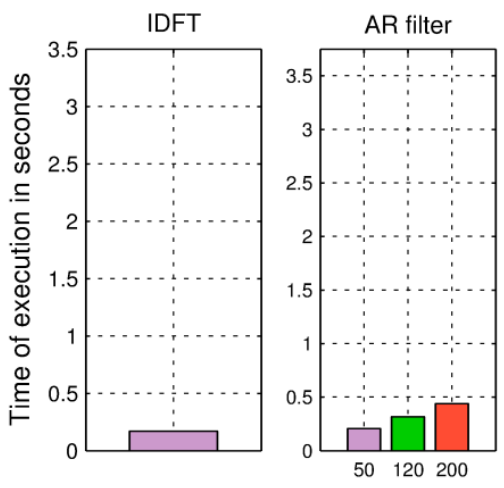

Filter order
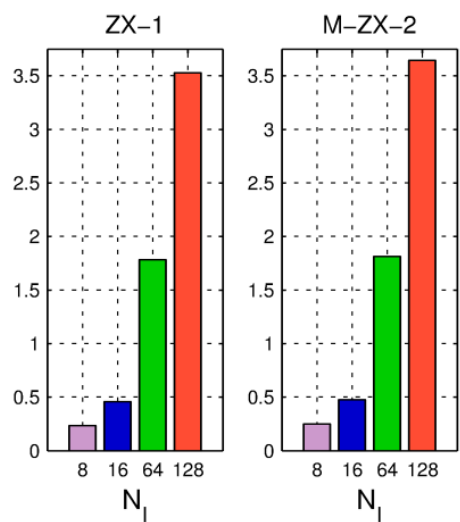

$\mathrm{N}_{\mathrm{I}}$

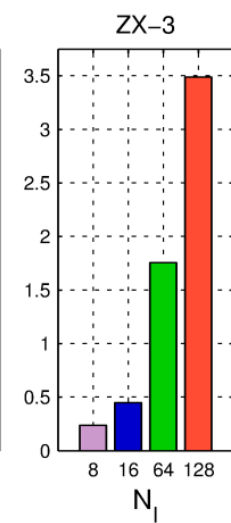

$\mathrm{N}_{1}$
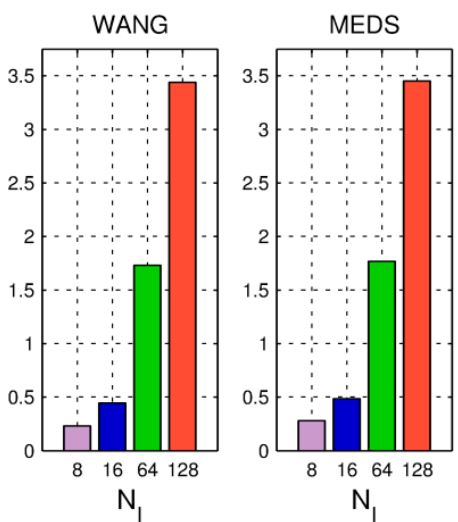

Fig. 3. Time to generate $10^{6}$ complex samples using the various Rayleigh fading generators.

It can be seen clearly that the smallest run time was obtained with the IDFT method due to the inherent efficiency of the FFT operation. The AR model was also quite efficient even for a high filter order. On the other hand, the SOS method appears to be most time-consuming method. All SOS-based models achieved approximately similar results with slightly better performance observed for the WANG model. For moderate $N_{I}\left(N_{I}=16\right)$, the WANG model has taken almost the same time as the AR model with 200th order filter, but with a loss of accuracy. The results presented in the previous section show that in terms of $G_{m e a n}, 64$ sinusoids are needed for WANG model to reach the performances obtained with 200th order AR filter.

\section{Conclusion}


In this paper, extensive computations have been conducted to analyze and compare seven of the most approved Rayleigh fading channel models. The recently published WANG model has shown good performances. Its second order statistics outperform those of the first Zheng and Xiao model (ZX-1) which has been considered for long time the most accurate among the SOS models. Although the MEDS achieves the best results in terms of the ACF of the in-phase component, we have shown that the WANG model outperforms by far the MEDS regarding the ACF of the complex envelope. Furthermore, WANG provides the best approximation of the $R_{g_{I} g_{Q}}(\tau)$ and it is a superior choice when a zero CCF is required (e.g., in the case of multiple uncorrelated fading waveforms). Moreover, the WANG model converges faster than the ZX-1 model and it needs a small number of simulation trials to achieve the desired statistical properties. For the Zheng and Xiao's SOS models, we deduce that ZX-1 stands as the best model as expected in some previous works. The comparison study shows that the well-known Young's IDFT method still has accurate statistical properties but due to its incapability to achieve sample-by-sample simulation, this method may be inappropriate for many applications. Finally, the simulation results demonstrate that the Badddour's AR FWGN model realizes the best accuracy in terms of the quality of the envelope PDF.

\section{References}

[1] Rappaport, T. S. (2002). Wireless Communications: Principles \& Practice (2nd ed.). Upper Saddle River, NJ: Prentice Hall PTR.

[2] Clarke, R. (1968). A statistical theory of mobile-radio reception. Bell System Technical Journal, 47(6), 957-1000.

[3] Xiao, C., Zheng, Y., \& Beaulieu, N. (2006). Novel sum-of-sinusoids simulation models for Rayleigh and Rician fading channels. IEEE Transactions on Wireless Communications, 5(12), 3667-3679.

[4] Young, D., \& Beaulieu, N. (2000). The generation of correlated Rayleigh random variates by inverse discrete Fourier transform. IEEE Transactions on Communications, 48(7), 1114-1127.

[5] Baddour, K., \& Beaulieu, N. (2005). Autoregressive modeling for fading channel simulation. IEEE Transactions on Wireless Communications, 4(4), 1650-1662.

[6] Jakes, W. (1974). Microwave Mobile Communications. New York: Wiley.

[7] Zheng, Y., \& Xiao, C. S. (2002). Improved models for the generation of multiple uncorrelated Rayleigh fading waveforms. IEEE Communications Letters, 6(6), 256-258.

[8] Patel, C., Stuber, G., \& Pratt, T. (2005). Comparative analysis of statistical models for the simulation of Rayleigh faded cellular channels. IEEE Transactions on Communications, 53(6), 1017-1026.

[9] Pätzold, M. (2012). Mobile Radio Channels (2nd ed.). Chichester, West Sussex, U.K.: Wiley.

[10] Wang, J., Ma, X., Teng, J., \& Cui, Y. (2012). Efficient and accurate simulator for Rayleigh and Rician fading. Transactions of Tianjin University, 18(4), 243-247.

[11] Skima, M. A., Ghariani, H., \& Lahiani, M. (2014). A multi-criteria comparative analysis of different Rayleigh fading channel simulators. AEU - International Journal of Electronics and Communications, 68(6), 550-560.

[12] Young, D., \& Beaulieu, N. (2003). Power margin quality measures for correlated random variates derived from the normal distribution. IEEE Transactions on Information Theory, 49(1), 241-252.

[13] Petrolino, A., Gomes, J., \& Tavares, G. (2008). A mobile-to-mobile fading channel simulator based on an orthogonal expansion. Proceedings of the IEEE Vehicular Technology Conference (pp. 366-370). Singapore.

[14] Stüber, G. (2002). Principles of Mobile Communication (2nd ed.). Norwell, MA: Kluwer Academic Publishers.

[15] McDonald, A. M., \& Olivier, J. C. (2007). A comparative study of deterministic and stochastic 
sum-of-sinusoids models of Rayleigh-fading wireless channels. Proceedings of the IEEE Wireless Communications and Networking Conference: (pp. 2027-2031). Kowloon, China.

[16] Press, W. H., Teukolsky, S. A., Vetterling, W. T., \& Flannery, B. P. (1992). Numerical Recipes in C: The Art of Scientific Computing. Cambridge, U.K.: Cambridge University Press.

[17] Deza, M., \& Deza, E. (2009). Encyclopedia of Distances. Berlin: Springer-Verlag.

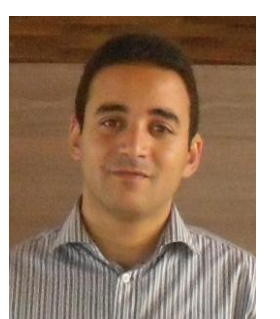

Mohamed Ali Skima was born in Tunis, Tunisia, in October 1979. He received the electrical engineering degree from the National Engineering school of Sfax (ENIS), Tunisia in 2003 and the electronic master diploma from the National Engineering School of Sfax (ENIS), Tunisia, in 2004. Currently, he is working toward the Ph.D. degree at Sfax University and he is a member of Laboratory of Electronics and Technology of Information (LETI). His research interests are modeling, characterization and sounding techniques of mobile radio channels.

Amira Boulaaba was born in Tunisia, in June 1978. She received the electrical engineering degree from the National Institute of Applied Sciences and Technology (INSAT), Tunisia in 2002. In 2003, she received the master degree in control engineering and industrial information technology from the INSAT, Tunisia. Crruntly, she is an associate professor at the Higher Institute of Technological Studies (ISET) of Rades, Tunisia. Her research interests are computer science, communication and wireless systems.

Hamadi Ghariani was born in Sfax, Tunisia, in July 1956. He received the electrical engineering degree from University of Sciences and Techniques of Sfax, Tunisia in 1981, the DEA degree in 1981 and his doctorate of engineer degree in 1983 in measurement and instrumentation from University of Bordeaux France. He joined the National Engineering School of Sfax since 1984. Crruntly, he is a professor in the same school. His research activities have been devoted to several topics include medical electronic, communication systems for medical telemetry, measure and instrumentation. 